\title{
Differential Diagnosis of Recurrent Hypersomnia: A Case Report of Primary Narcolepsy and Acute Transient Psychotic Attack
}

\author{
(D) Joanna Maria Kıyak1, (1) Sezen Köse2, (1) Melek Hande Bulut Demir³, (1) Burcu Özbaran² \\ 1'zzmir Katip Çelebi University, Atatürk Training and Research Hospital, İzmir, Turkey \\ ${ }^{2}$ Ege University Faculty of Medicine, Department of Child and Adolescent Psychiatry, İzmir, Turkey \\ ${ }^{3}$ Behçet Uz Children Hospital, İzmir, Turkey
}

\begin{abstract}
Narcolepsy is a childhood and adolescence disorder, that until recently remained unidentified until adulthood, with a reported time from onset to diagnosis of around a decade. This disorder affects approximately $0.05 \%$ of the population and starts in childhood and adolescence about half of the time. The main symptom of hypersomnia is excessive daytime sleepiness or prolonged night-time sleep that has occurred for at least 3 months prior to diagnosis. Patients with narcolepsy also exhibit hallucinations and delusions, however the differential diagnosis between the disorders is often unclear. Case presented in our study is an example of atypical narcolepsy with coexistence of psychotic symptoms. In our case upon the set-off of the second attack of excessive daytime sleepiness, the differential diagnosis of recurrent hypersomnia was not considered. This is due to the fact that the first attack of narcolepsy with hallucinatory component of the patient could have been misdiagnosed as acute transient psychotic attack. Hallucinations and delusions sometimes appear in patients with narcolepsy. The differential diagnosis between the disorders is not always simple. In our study we present the case which illustrate this overlap. Child and adolescent patients, which demonstrate psychotic symptoms and excessive daytime sleepiness component, should be concidered in case of evaluation for a diagnosis of primary hypersomnia following a multidisciplinary cooperation of neurologists, paediatricians and psychiatrists.
\end{abstract}

Keywords: Narcolepsy, primary hypersomnia, excessive daytime sleepiness, psychotic symptoms

\section{Introduction}

Narcolepsy is a childhood and adolescence disorder, that until recently remained unidentified until adulthood, with a reported time from onset to diagnosis of around a decade. This disorder affects approximately $0.05 \%$ of the population and starts in childhood and adolescence about half of the time (1).

The main symptom of hypersomnia is excessive daytime sleepiness (EDS) or prolonged night-time sleep that has occurred for at least 3 months prior to diagnosis.
Hypersomnia can be primary (of central/brain origin) or secondary to a number of medical conditions. However, more than one type of hypersomnia can coexist in a single patient. The true primary hypersomnias include narcolepsy (with or without cataplexy), idiopathic hypersomnia and recurrent hypersomnias (such as Klein-Levin syndrome).

Secondary hypersomnias are numerous. Hypersomnia can be secondary to disorders such as clinical depression, multiple sclerosis, encephalitis, epilepsy or obesity. Hypersomnia can also be a symptom of other sleep 
disorders such as sleep apnea. It may occur as an adverse effect of the taking or withdrawal of certain medications, or of drug or alcohol abuse. A genetic predisposition may be a factor for the development of hypersomnia. In some cases, hypersomnia results from a physical condition, such as a tumour, head trauma or dysfunction of the autonomic or central nervous system (2).

According to the DSM-5, narcolepsy is a disorder in which the individual will experience recurrent periods of an irresistible need to sleep, or will fall asleep, or nap within the same day, regardless of whether or not the time and place are appropriate. These episodes must occur at least three times per week over the prior three months, accompanied by at least one of the following symptoms: episodes of cataplexy, or loss of muscle tonus, occurring several times a month, episodes lasting seconds to minutes of sudden full body loss of skeletal muscle tone without Loss of Consciousness (LOC) that are precipitated by an episode of strong emotion, or objective measure of hypocretin deficiency in the cerebrospinal fluid. Laboratory test results must reveal hypocretin-1 immunoreactivity equal to or less than one-third of hypocretin-1 found in healthy subjects (alternatively, equal to or less than $110 \mathrm{pg} / \mathrm{mL}$ ). In addition, nocturnal sleep polysomnography (PSG) study should reveal abnormally low rapid eye movement (REM) sleep duration ( $<15$ minutes inclusive) or a multiple sleep latency test (MSLT) showing a mean sleep latency $<8$ minutes, and $>2$ REM sleep episodes upon the onset of sleep (3).

Patients with narcolepsy also exhibit hallucinations and delusions, however the differential diagnosis between these disorders is often unclear.

A number of patients with narcolepsy have also experienced hypnagogic or hypnopompic hallucinations, which were possibly misdiagnosed as schizophrenia due to the similar symptomatology (4). However, the association between narcolepsy and schizophrenia remains inconclusive. A review article suggested that comorbidity of narcolepsy and schizophrenia is likely to be rare and sporadic (5).

We report a case of a 17-year-old boy with recurrent EDS, which was first noticed 2 years before the diagnosis, and subsequently noted again the following year in the same season. During the first episode of excessive sleepiness, the patient was experiencing psychotic symptoms such as delusions of reference and persecution, as well as visual and acoustic hallucinations. In the second episode, negative psychotic symptoms were observed.

\section{Case Report}

$Y$. is a 17-year-old boy. He is the first and only living child of a 31-year-old house wife mother and 37-year-old farmer father. Y.'s mother and father are first degree cousins. The birth of the patient was through a planned caesarean section, both pregnancy and delivery were unremarkable. There is nothing out of ordinary in the patient's birth and postnatal history. The patient was healthy and reached developmental milestones within normal ranges. He was raised by his mother and grandmother at home and did not attend nursery. When Y. was 2 years old, his 3-month-old sister died of febrile convulsion. When he was 3 years old, he suffered from measles. At the age of 4 , he was operated on due to inguinal hernia.

No unusual behaviour or problems were noted until the patient began secondary school (at 15 years of age) and his parents observed that he became internally preoccupied and inattentive. Before the excessive sleepiness started, the patient was seen by a physician because of flu-like symptoms. The physician prescribed anti-flu medications and the patient recovered in one week. Subsequently, the patient started exhibiting social withdrawal, suspiciousness, inattentiveness, excessive sleepiness and the tendency to fall asleep in public places. At first, the patient had difficulty waking up in the morning to go to school and he became drowsy at school. His academic performance became poor and he failed to attend school because of increasing sleepiness. Approximately 2 months after the onset of excessive sleepiness, he started experiencing psychotic symptoms including delusions of reference and persecution, as well as visual and acoustic hallucinations. Both of these symptoms (EDS and psychotic symptoms) lasted for approximately 4 months, during which the average daily sleep period during the episodes was 20 hours.

Y. was referred to our psychiatry department. The main reason for this referral was the change in the boy's behaviour. This change in the patient's attitude was first noticed 4 months before the referral and he exhibited no response to a $500 \mathrm{mg}$ valproic acid per day and 10 mg fluoxetine per day medication programme that was prescribed in the outpatient clinic. When the patient was referred to our clinic, excessive sleepiness was no longer observed. He had no unusual physical or neurological features. Standard laboratory tests were carried out, including diffusion magnetic resonance imaging (MRI), antinuclear antibodies, C3, Lyme and Brucella tests, all of which were reported within the normal range. The patient's awake electroencephalography (EEG) showed slow wave pattern in basic rhythm, whereas sharp wave paroxysmal 
activity was detected in the frontal hemispheres. Y. was diagnosed with acute transient psychotic attack. The patient was treated as an in-patient with medication consisting of $2 \mathrm{mg}$ risperidone per day. Y. showed sustained clinical and functional improvement, which was noticeable soon after the administration of risperidone. After 1 month of treatment in our psychiatry clinic, the patient was discharged and he returned to his premorbid condition. The patient underwent a Wechsler Intelligence Scale for Children test that was performed in a symptom free period yielding IQ scores indicating mild mental retardation.

Y. was free of symptoms for 22 months following discharge from the psychiatry clinic. During this symptomfree period, he was administered $1 \mathrm{mg}$ risperidone per day and episodes of hypersomnolence were not noticed. A few weeks prior to the second psychiatric referral, the patient was seen by a physician due to complaints of apathy and sleepiness. The patient was prescribed anti-flu medications. This coincided with the onset of excessive sleepiness. The patient's daily average sleep in these episodes was 20 hours. $Y$. was again referred to our clinic and was treated as an in-patient for a range of symptoms he was suffering from: hypersomnia, long unsatisfactory naps, difficulty waking up from naps, sleep related cognitive problems (concentration problems, poor blurry memory), drowsiness and a diminished sense of well-being. The patient's symptoms restricted him from engaging in normal social activities. The patient did not have sleep paralysis, snoring was not observed, and he did not exhibit symptoms indicative of cataplexy. During the first interview, his cooperation was unsatisfactory, his mood was depressive and irritable, he displayed flat affect and he gave short answers consisting of "yes" or "no". He was not disorientated in the sense of time, direction, and recognition of people or places. He was aggressive, showing psychomotor retardation, however, he did not have suicidal thoughts nor was he experiencing positive psychotic symptoms. The patient also did not exhibit abnormal eating patterns, an increase in sexual interest/masturbation or absurd behaviour. In the Clinic Global Severity scale, he was given 5 points. During the first 2 weeks of the in-patient treatment, he was sleeping for approximately 15-20 hours per day. When the patient was awake, he was unhappy and tired. At the beginning of his in-patient treatment, he became agitated and was disinhibited. During the interview, he started swearing and was uncooperative. After that incident, $15 \mathrm{mg}$ olanzapine per day and $7.5 \mathrm{mg}$ lorazepam per day were added to his $3 \mathrm{mg}$ risperidone per day treatment.

Standard laboratory tests were carried out, including blood count, biochemistry (liver and kidney parameters) and endocrinology parameters. Aspartate aminotransferase 84 and alanine aminotransferase 147 were reported. Due to an increase in transaminase levels, the patient was referred to the Child Gastroenterology Department. Upon physical examination and abdomen ultrasonography, hepatomegaly was observed. Since portal doppler ultrasonography was indicative of portal hypertension, the patient was transferred to the Child Gastroenterology Department for further examination. Differential diagnosis of chronic liver disease (autoimmune hepatitis/Wilson disease) was made and a liver biopsy was taken. Serologic examination for hepatitis A virus, hepatitis B virus, hepatitis C virus, hepatitis E virus, cytomegalovirus, Rubella, and Brucella was negative. Control portal doppler ultrasonography did not show any pathology typical for chronic liver disease. The patient's liver biopsy was normal.

The patient was referred to Child Neurology to investigate the cause of the hypersomnia. Diffusion brain MRI was normal. His awake EEG, limbic encephalitis, quantitative urine-blood amino acid chromatography (TANDEM) and homocysteine were reported to be in the normal range. Neurologic aetiology was not detected. Our patient was referred for a screening PSG sleep study. The initial sleep study revealed increased REM sleep latency (43\% of total sleep time). The result of the PSC study was abnormal and suggestive of narcolepsy. Sleep-disorder breathing was not identified. Finally, a MSLT was performed to objectively quantify the degree of sleepiness. This test consisted of 5 naps opportunities, given at 2-hours intervals. His MSLT showed an overall mean sleep latency of $1 \mathrm{~min}$. His mean REM latency was 1.7 min, and sleep-onset REM was observed 3 times. Between the tests, he had no episodes of cataplexy, hence the patient was diagnosed with narcolepsy without cataplexy. We found that the mixed symptoms of narcolepsy and psychotic attack improved with antipsychotic agent treatment. Y. showed sustained clinical and functional improvement, which was noticeable soon after the administration of risperidone. After 2 months of treatment in our psychiatry clinic, the patient was discharged with a medication programme of $1 \mathrm{mg}$ risperidone per day. He returned to his premorbid condition and so far has not experienced either EDS attack or psychotic symptoms. The approval concerning publication of case's treatment and follow up period was obtained from patient's parents.

\section{Discussion}

The case presented in our study is an example of atypical narcolepsy with the coexistence of psychotic symptoms. The patient's psychotic symptoms were especially prominent 
during the first attack. The EDS period was followed by psychotic symptoms including delusions of reference and persecution, as well as visual and acoustic hallucinations. However, during the second attack, negative psychotic symptoms were more prominent.

Y. was first admitted to our department with a diagnosis of a psychotic attack. After he was prescribed risperidone therapy, psychotic symptoms diminished and the patient was diagnosed with acute transient psychotic attack. As there was no excessive sleepiness when $Y$. was first admitted to our department, primary hypersomnia was not considered as a differential diagnosis.

In our case, upon the onset of the second attack of EDS, the differential diagnosis of recurrent hypersomnia was not considered. This is due to the fact that the first attack of narcolepsy with hallucinatory component of the patient could have been misdiagnosed as acute transient psychotic attack.

Hallucinations and delusions sometimes appear in patients with narcolepsy. The differential diagnosis between these disorders is not always simple. Here we present examples which illustrate this overlap.

Mlynczak (6) reported that $17 \%$ of psychotic patients suffered from a variant of narcolepsy. Saucerman suggested that some patients diagnosed with schizophrenia or other psychotic disorders may actually be suffering from a variant of narcolepsy with a genetic background involving dominant hypnagogic hallucinations (7). Narcolepsy in which a hallucinatory component is unusually prominent is rare, but has been previously described and may lead to the development of an illness indistinguishable from the Schizophrenic syndrome. Takeuchi et al. (8) described a case of a narcoleptic female patient whose delusions and hallucinations appeared at diagnosis of narcolepsy. Her psychotic state improved with anti-psychotic medication (8). Royant-Parola (9) found that nearly half of 11 narcolepsy patients manifested a psychiatric disorder, in which a hallucinatory component was central, leading to a misdiagnosis of schizophrenia. However, all of the above cases consider adult patients, whereas the coexistence of narcolepsy and psychotic syndromes in children or adolescents has been reported just twice in the case report study of a 13-year-old patient suffering from comorbidity of childhood-onset narcolepsy and adolescent-onset schizophrenia (9) and the case report of a 14-year-old girl who developed narcolepsy with cataplexy and a psychosis during adolescence (10).

In reviewing the previous literature, three possible differential diagnoses were found that could explain the comorbidity (11-14). First, narcolepsy may co-occur by chance with schizophrenia or other psychiatric disorders. Second, some authors support the existence of a psychotic form of narcolepsy, in which the psychotic symptoms exceed the common hypnagogic or hypnopompic hallucinations. Third, psychotic symptoms develop sequentially after treatment with central stimulants (i.e., methylphenidate and modafinil). Although they might be difficult to differentiate, psychotic symptoms differed between patients with narcolepsy and schizophrenia $(4,11,12,14)$. Previous studies demonstrated that narcoleptic patients experience multisensory hallucinations to a significant extent, and not just the predominantly verbal-auditory hallucinations of schizophrenic patients. Delusions and associated delusional behaviour are rarely observed in patients with narcolepsy. Although the psychotic-like symptoms of narcoleptic patients (sleep-associated hallucinations) differ from the core symptoms of schizophrenia (delusions, disorganized behaviour, and negative symptoms) the differential diagnosis between the disorders is often unclear $(4,15)$.

Our patient did not exhibit any comorbidities that would match with secondary hypersomnia. The initial sleep study did however reveal increased REM sleep latency (43\% of total sleep time). The result of the PSG study was abnormal and suggestive of narcolepsy. Finally, a MSLT was performed to objectively quantify the degree of sleepiness. As a result of the above examinations, the patient was diagnosed with primary hypersomnia-narcolepsy without cataplexy. We found that the mixed symptoms of narcolepsy and psychotic attack improved with anti-psychotic agent treatment.

Child and adolescent patients who demonstrate psychotic symptoms and EDS component should be evaluated for a diagnosis of primary hypersomnia following a multidisciplinary cooperation of neurologists, paediatricians and psychiatrists. Additionally, a carefully scrutinized clinical assessment and a sleep test can help clinicians make a proper differential diagnosis.

\section{Ethics}

Informed Consent: The approval concerning publication of case's treatment and follow-up period was obtained from patient's parents.

Peer-review: Externally peer-reviewed.

\section{Authorship Contributions}

Concept: J.M.K., S.K., B.Ö., M.H.B.D., Design: J.M.K., Data Collection or Processing: J.M.K., M.H.B.D., Analysis or Interpretation: J.M.K., S.K., Literature Search: J.M.K., S.K., Writing: J.M.K. 
Conflict of Interest: No conflict of interest was declared by the authors.

Financial Disclosure: The authors declared that this study received no financial support.

\section{References}

1. Sullivan SS. Narcolepsy in adolescents. Adolesc Med State Art Rev 2010; 21:542-55.

2. Dauvilliers Y. Differential diagnosis in hypersomnia. Curr Neurol Neurosci Rep 2006; 6:156-62.

3. Association AP. Diagnostic and statistical manual of mental disorders (DSM- $5^{\circledR}$ ). 5th ed. American Psychiatric Pub; 2013.

4. Fortuyn HAD, Lappenschaar GA, Nienhuis FJ, et al. Psychotic symptoms in narcolepsy: phenomenology and a comparison with schizophrenia. Gen Hosp Psychiatry 2009; 31:146-54.

5. Walterfang $M$, Upjohn E, Velakoulis D. Is schizophrenia associated with narcolepsy? Cogn Behav Neurol 2005; 18:113-8.

6. Mlynczak ST. Screening of psychotic patients for the schizophreniform variant of narcolepsy. Master Thesis. University of Alberta, Edmonton Kanada, 1993.

7. El Omari F, Salomonsen-Sautel S, Hoffenberg A, Anderson T, Hopfer C, Toufiq). Prevalence of substance use among moroccan adolescents and association with academic achievement. World J Psychiatry 2015; 5:425
8. Takeuchi N, Mukai M, Uchimura N, Satomura T, Sakamoto T, Maeda $\mathrm{H}$. A narcoleptic patient exhibiting hallucinations and delusion. Psychiatry Clin Neurosci 2000; 54:321-2.

9. Royant-Parola S. [Narcolepsy and the psychiatric tableau. A specific form of narcolepsy?] Ann Med Psychol (Paris) 1992; 150:286-90

10. Canellas-Dols F, Delgado C, Arango-Lopez C, Peraita-Adrados R. Narcolepsy-cataplexy and psychosis: a case study. Rev Neurol 2017; 65:70-4

11. Douglass AB. Narcolepsy: differential diagnosis or etiology in some cases of bipolar disorder and schizophrenia? CNS Spectr 2003; 8:120-6.

12. Kishi $Y$, Konishi S, Koizumi S, Kudo Y, Kurosawa H, Kathol RG. Schizophrenia and narcolepsy: A review with a case report. Psychiatry Clin Neurosci 2004; 58:117-24.

13. Kondziella D, Arlien-Soborg P. Diagnostic and therapeutic challenges in narcolepsy-related psychosis. I Clin Psychiatry 2006; 67:1817-9.

14. Talih FR. Narcolepsy presenting as schizophrenia: a literature review and two case reports. Innov Clin Neurosci 2011; 8:30-4.

15. Dahmen N, Kasten M, Mittag K, Müller MJ. Narcoleptic and schizophrenic hallucinations. Eur J Heal Econ 2002; 3:94-8. 